\title{
SMART MAINTENANCE FrAMEWORK BASED ON SPATIO-VISUAL COMPUTING: The CASE OF AirCRAFT MRO
}

\author{
Polina Bortsova \& Peter Panfilov
}
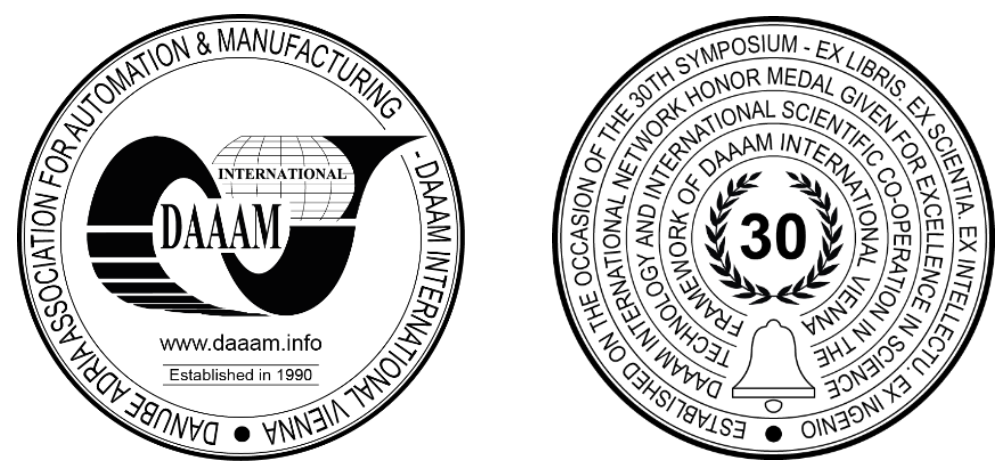

This Publication has to be referred as: Bortsova, P[olina] \& Panfilov, P[eter] (2021). Smart Maintenance Framework Based on Spatio-Visual Computing: The Case of Aircraft MRO, Proceedings of the 32nd DAAAM International Symposium, pp.0673-0684, B. Katalinic (Ed.), Published by DAAAM International, ISBN 978-3-902734-33-4, ISSN 1726-9679, Vienna, Austria

DOI: $10.2507 / 32$ nd.daaam.proceedings.095

\begin{abstract}
Many organizations in aviation industry are facing the problem of establishing an overall coherent picture of their business and operations, while disparate datasets from different departments and geographically dispersed locations are united only by experience and often-heterogeneous hardware and software platforms prevent from efficient enterprise application integration. This paper focuses on approaches to increasing the value proposition of management information systems of aviation organization through integrated spatial and visual computing solutions while using the domain of aircraft maintenance, repair and overhaul organization as an illustrative example. The subject of the research is the analysis of the application of new computing approaches to digital transformation of complex geographically distributed services that require timely coordination, accurate localization and efficient planning and execution. The main object of the study is a new integrated spatio-visual framework for smart MRO service of aircraft engines that includes technology and software solutions in support of operations at different levels in aviation organization from top management personnel to the aircraft technician.
\end{abstract}

Keywords: aircraft MRO; spatial computing; visual computing; augmented reality.

\section{Introduction}

In the last decade, visual computing is under intensive study in a wide range of application areas. In particular, augmented reality (AR) as a subarea of visual computing has received special attention as an advanced approach to improve the user interface at the level of management information system (MIS) architecture both in the industrial sector and in the sector of providing various services. The visual computing interfaces can be designed for different kinds of users, and support several objects and applications in several areas, depending on the specifics of the system being built. Spatial computing is a new computing paradigm driven by advances in location-aware smart applications and services, machine learning (ML) and the Internet of Things (IoT) that enables seamless interactions between people, machines and environments to optimize processes in real or near real time. Spatial computing acts as a mean that allows people to understand better, how machines, products and process participants move in space, and what relationships exist between them and how all the information related to their location can be used for business processes. 
Location Intelligence (LI) is achieved through visualization and analysis of geospatial data, enhancing the ability to make decisions and predictions. The main goal of the presented study is to consider approaches to unlocking the potential of spatial and visual computing by suggesting an integrated spatio-visual framework that integrates existing geospatial solutions into the future intelligent transportation systems (ITS). We use an example of aviation industry and a specific sector of aircraft engine maintenance, repair and overhaul (MRO) service as a business case for the framework prototyping and demonstration.

\subsection{Digital transformation of transportation sector}

Intelligent Transportation Systems (ITS) provide solutions to the current transportation problems with the help of new digital technologies and computing paradigms. ITS is a "smart" system that uses innovative developments in the modelling of transport systems and the regulation of traffic flows, providing end users with greater information content and safety, as well as qualitatively increasing the level of interaction of transportation system users compared to conventional transportation systems. The overall idea behind the ITS is to improve decision making, particularly in real time, by vehicle operators, transport network controllers and other users, thereby improving the operation and safety of the entire system. ITS is an integrated system that implements a broad range of computing, communication, control, vehicle sensing and electronics technologies to help in monitoring and managing vehicle status, traffic flow, enhancing productivity of the system, and saving lives, time and money. Emergency Management System (EMS) is the newest research field in ITS domain. EMS is mainly concerned with the application of different digital technologies to develop a transport system that can provide help in the emergency conditions. It can help in reducing the fatality rate in the accidents [1].

Achieving mobility, safety and sustainability are in focus of ITS research and development. Although significant progress has been made in visual computing techniques such as computer vision in ITS, researchers from both academia and industry are still facing several major challenges that hinder further advances in ITS development that are represented with the following How questions:

- How to improve the visual perception for ITS in adverse weather conditions?

- How to take full advantage of multi-sensor perceptual data in ITS?

- How to develop the ITS-specific visual computing solutions through advanced artificial intelligence techniques?

- How to develop a visual computing-based traffic monitoring system and enhance the situational awareness and safety?

- How to guarantee the effectiveness of different autonomous vehicles using visual computing techniques?

There exists a broad spectrum of technologies intended to support applications and systems developed for ITS. The scope of modern technologies and possibilities of their fully integration in a typical smart transportation service project are going to be discussed in in this work.

\subsection{Digital transformation of aviation transportation: Aircraft health monitoring example}

Although avionics and control technologies have produced continuous advances in aircraft systems, there is still significant opportunity for greater efficiency, enhanced functionality, and better integration of aviation systems. This is particularly true for systems that reduce the burden on the crew of flying the aircraft and systems that allow for increased capacity of the global air traffic management (ATM) system. Using big data and data analytics together with secure communications capabilities opens up a host of possibilities to monitor an aircraft's components and on-board equipment status. Real-time data enables aircraft operators to apply predictive maintenance to monitor broken parts. Moreover, data analytics can improve fuel usage, as fuel is one of the biggest expenses for airlines. The big challenge, however, is extracting relevant data and making it useful, i.e. transforming airline operations data from maintenance to flight safety in the end-to-end manner [2]. More and more value is being extracted from aircraft data, delivering intelligence and analysis that are able to transform operations. Data can be unlocked from every part of the aircraft - cockpit, cabin, aircraft engine, and used for enhancement of performance and service. Accessing this data is rather complex and that is why effectiveness of handling data is playing a key role in digital transformation of aviation transportation.

Current state of the aviation industry requires new digital "smart" solutions [3], because the majority of reporting, diagnosis, planning and maintenance services happen on ground. Due to aircraft and engine condition issues, costly delays and cancellations of transportation services occur and, consequently, airplane serviceability is interrupted. Aviation industry experts and specialists are increasingly discussing issues of big data technologies, data ownership, access, and management in relation to the aircraft health monitoring (AHM), as well as the benefits and challenges for airlines, aircraft OEMs, maintenance, repair and overhaul (MRO) organizations, and the role of each player in the AHM market. More and more data from aircraft engines, airplanes, and on-board avionics systems is available for the measurement, storage, and transmission that require a new way of operating aircraft — a connected and integrated operation that brings increased efficiency for airlines [4]. 
Aircraft engines are the most expensive part of an aircraft, and the carrier is interested in keeping its power plants in good condition. In addition to the fact that the engines are important for fast and safe flight, unscheduled maintenance due to problems can become very expensive. The most expensive parts of the engine are the control systems, turbine blades and bearings. Prices for aircraft power plants range from \$12 million to \$45 million. Despite the fact that they are made of the most modern and durable materials, jet engines cannot avoid wear and tear. This occurs because of vibration, friction, high temperatures and corrosion from external influences or damage from foreign objects. High speed, high temperature, high load of work environment contribute to corrosion, wear and failure condition. Aircraft engine maintenance standards are the most important thing in ensuring flight safety and have a huge impact on the financial condition of airlines around the world. Answering the question, why do accidents happen, statistics provide the figures that highlight above all human error factor and situations when industrial equipment (engines in particular) is elevated or is hard to access.

Many airlines partially or fully outsource MRO services from repair shops and system suppliers. MRO software manages the maintenance process and offers solutions to improve efficiency and enhance the end-user experience. Airlines require software that will optimize productivity and keep operations up with demand. New technologies and the digitalization of services are creating benefits for airline and MRO operators to streamline maintenance, reduce operating costs, and take full advantage of the skyrocketing air travel demand. Key factors such as rising demand for air travel increase in digitalization and upgrade of the legacy MIS to digital MRO Software influence growth within the MRO Software market. Most of leading manufacturers of aircraft engines invest into digital transformation of their businesses including engine production, sells, repair, and maintenance services (Table 2).

\begin{tabular}{|l|l|}
\hline \multicolumn{1}{|c|}{ OEM } & \multicolumn{1}{c|}{ Digitalization initiative } \\
\hline GE & $\begin{array}{l}\text { Flight efficiency/operational management } \\
\text { Fuel management } \\
\text { Predictive maintenance } \\
\text { Fleet management }\end{array}$ \\
\hline Rolls-Royce & $\begin{array}{l}\text { Analytics service into Total Care for analysing engine data in real-time } \\
\text { Managing customers' engine maintenance } \\
\text { Monitoring aircraft availability }\end{array}$ \\
\hline Pratt \& Whitney & $\begin{array}{l}\text { Data ecosystem } \\
\text { System for capturing thousands of engine and aircraft parameters } \\
\text { throughout the full flight cycle } \\
\text { Monitoring engine performance for minimization of disruptions }\end{array}$ \\
\hline
\end{tabular}

Table 1. Engine manufacturers' digitalization initiatives

\subsection{Research goals and tasks}

The complexity and difficulty of aviation transportation management has increased due to the wide range of business areas and departments involved in aviation transportation business. Within the framework of this research, the main interest is put to the information flow between the parties of the organizational process of the aviation transportation service as it is provided in the Figure 1. The distributed connected (networked) structure of the aviation transportation organizations and their operations implies that in case of digital transformation of aviation services real time exchange of data and the distributed nature of the MIS in organization are important requirements and prerequisites.

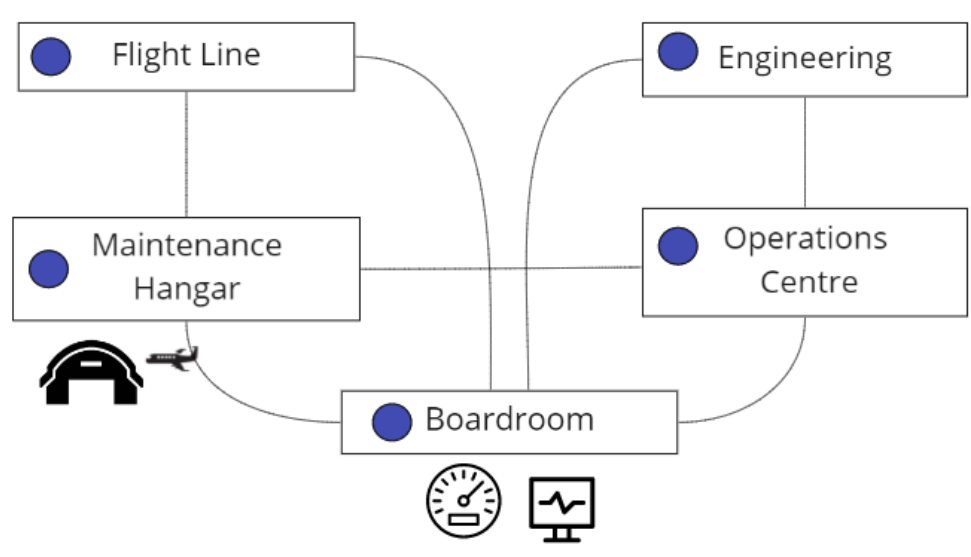

Fig. 1. Key elements of the aviation transportation service organization. 
The Figure 2 provides the ICF Aviation [5, 6] described a high-level conceptual view of the AHM practice as it. Elements of advanced digital technologies of the smart MRO service application should address the issues during health checks and effective communication between OEM Tech Support, Airline Maintenance Control Centre, Aircraft and Maintenance Engineering Operations. The position of each instance relative to each other as well as full description of the common information with regular updates, parallel processing of spatial data, visualization of geolocations of the aircrafts and conditions of all systems play a decisive role in AHM performance improvement [7, 8]. Key factors such as rising demand for air travel increase in digitalization and upgrade of the legacy MIS to digital smart MRO influence growth within the MRO Software market.

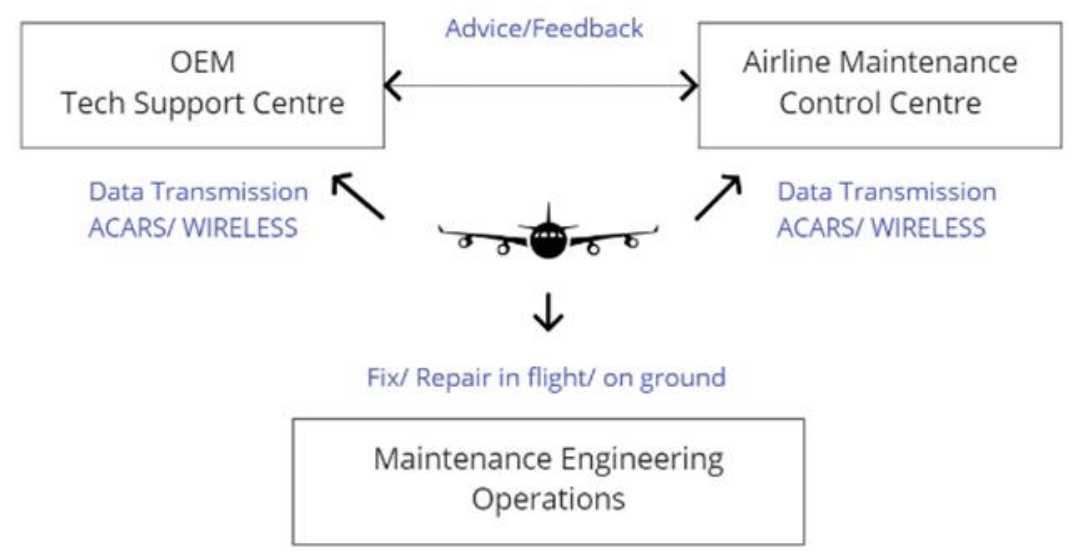

Fig. 2. Aircraft health monitoring schematic.

Based on the analysis of existing digitization initiatives [5] and the research on aircraft MRO topic the following forms of initiatives that can be supported by the suggested smart maintenance framework were formulated:

- Transformation of technical services

- Automated and secure transparent system of digital reporting on work with components of aircraft

- Engine monitoring based on the processing of an engine status information collected from sensors

- Building a model for accurate prediction of the remaining useful lifetime (RUL) and optimal scheduling of the engine replacement/repair service with exact location tag

- Profound data analytics, a work with historical data for mining and collecting insight to enhance operations and reduce maintenance delays

- Collecting fleet data for maximization of the customer's specific engine performance and time-on-wing while maintaining predictable MRO

- Spatial computing bolsters interaction of workers with the physical systems in industrial environments

- Full usage of Visual Computing potential.

Supposedly, in future, the customer will support the maintenance company or the machine manufacturer over long distances with the repair of the machine via live transmission. The customer will need a high-speed Internet connection and data glasses, and the manufacturer a PC. With the benefits of innovative Visual Computing technologies, the aviation technicians equipped with AR data glasses will have a projection surface in front of their eyes that will show different files: images, videos, and sound or text document. These displays overlay and complement the real image, i.e. the view of the equipment to be serviced or repaired. The repair and maintenance costs will therefore decrease; long journey times for the service technician, as well as machine downtime and production failure at the customer side will be outdated.

In this section, the application of the proposed architecture to the solution of aviation transportation problems is considered given the example of, particularly, aircraft engine maintenance, repair and overhaul (MRO) smart service [7, 8]. Firstly, the subject area, environment, key participants and level of current digitalization as well as the scope of upto-date technologies used by manufacturers are analysed. Then, possible implementation of the proposed framework from the previous section, enriched with specific recommendations, is discussed.

The aircraft engines MRO was chosen in this work as a specific illustrative example for detailed analysis and implementation of the application framework. The MRO in aviation is the repair, service, or inspection of an aircraft or aircraft component(s). It is applicable to all maintenance activities that are conducted to ensure safety and airworthiness of all aircrafts by international standards. Safety of aircraft is influenced by the condition of the core component of the aircraft; it is needed for OEMs and airlines to understand permanently, whether engine could be operated normally. 
The modern digital technologies such as spatial and visual computing can offer desired Smart MRO solutions that could lead to minimization of risks that threaten safety in the air, for instance, by giving an opportunity to track the location of engines in the sky, building digital twins for them and developing new approaches and tools for engine maintenance.

To achieve this goal, the authors have identified a number of tasks to be solved on the way to the design and implementation of the universal spatio-visual computing framework:

- Discussion of spatial and visual computing areas, as well as their interaction and comparison

- Analysis of the possibilities of practical application of these technologies to aviation transportation problems

- Design of universal integrated framework for supporting information systems, combining advanced related technologies: AR, digital twins, GIS, geographic positioning systems (GPS), data analysis, ML

- Considering the application of spatial and visual computing within the proposed framework for the example application of aircraft engine MRO.

\section{Methodology}

For the study, methods of synthesis of information on the selected topic, deduction, critical analysis of technologies, their comparison and search for complementary components were used.

\subsection{Spatial computing}

Digital transformation includes a network of interconnected processes and solutions. To transfer value of the network it is necessary to provide its transparent visibility of the connections, incl. in physical space. As part of the digital transformation, many organizations rely on geographic information systems (GIS) technology to generate location information. The technology has recently expanded and now it includes advanced spatial computing capabilities. Spatial computing ranks as one of the 10 most promising technologies of 2020 according to the World Economic Forum's report in terms of the value of their potential contribution to the transition to Industry 4.0 [9]. More than 90\% of companies believe that location information is critical for achievement of business success. Geospatial data and spatial analysis of location are used to generate the knowledge needed to make decisions. Applications of this nature can be used to find the best places to select a marketplace and solve bottlenecks on the roads, maintain and repair vital infrastructures. In computer science, MIT Media Lab alumnus Simon Greenwold in his futuristic dissertation in 2003 firstly defied the concept [10]. Spatial computing is primarily the ability to relate the location in space of one object to its operational control or its virtual complement elsewhere. From a practical point of view, spatial computing is the digitization of the actions of machines, people, objects and the environment in which they are located in order to ensure and optimize their actions and interactions. The key difference between traditional and spatial computing is the orientation of spatial computing to the three-dimensional location of the object in question [11].

Technically speaking, spatial computing is a technology that uses software to seamlessly integrate user interface that provides human-computer interaction into the 3D physical world. In the literature devoted to the study of spatial computing the following areas of knowledge are considered for studying:

1. The science of spatial computing that cover among others the problems of spatio-temporal prediction, working with qualitative spatio-temporal data and development of spatial and spatio-temporal computing standards.

2. Spatial computing systems issues include definition and development of spatial computing infrastructure; collection, integration and storing sensed data; computational aspects of spatial Big Data, and augmented reality.

3. Crosscutting solutions focus on ubiquitous computing arrangements, sustained sensing and monitoring, reliable transportation systems and geo-confidentiality.

Technologies. The main approach in spatial computing is to enable the real world to interact with digital entities. Thanks to a smart combination of technologies such as AR, which provides an interface between a digital world and a physical one, the industrial Internet of Things (IoT), which is responsible for the control of products and assets, machine learning, sensor technologies, a large number of processes in logistics and production, can be optimized. Due to virtual and augmented reality, the concept of the digital twin in spatial commutation is becoming extremely valuable. Digital twin [9] refers to a digital copy or model of a physical twin: processes, people, places, systems and devices that can be used for various purposes, such as modeling, predicting behavior, monitoring performance, restoring an object for 3D printing, checking scenarios, and workouts, connecting to other objects in the digital space. 
To create a digital world, software algorithms (such as methods for optical measurement of distance or speed, video recording, and other geolocation technologies) integrate the digital map with sensor data and digital representations of objects and people to purposefully influence the real world. A physical object has IoT sensors that constantly collect various forms of data and transmit information to a digital twin to analyze and perform the above functions. The results obtained with the virtual models can be applied further to the physical twin.

In terms of architecture, spatial computing provides a great opportunity for scripting, giving a possibility and access to generating plenty of ideas, especially for dynamic spaces such as rooms, buildings, neighborhoods, cities, and everything that populates these spaces. Spatial computing and augmented reality together enable architecture with an ideal and realistic view of the space available and the "virtual lens" mechanism through which new information can be viewed and applied.

Application areas. One of the most visible aspects of spatial computing are its analytical capabilities. For instance, using spatial heat maps, companies can obtain the following information: where workers are located most often during their work process, where unexpected machine downtime occurs, which routes are most frequently used. Additionally, the considered approaches can provide safety. With the help of augmented reality within a factory, safety zones and routes can be made for both people and cars. Spatial computing helps to understand the user's location, find the surrounding elements in space and give directions on the way to a right safe place using AR. During an emergency evacuation, the spatial computing can help to find the most efficient exit route for each individual. Information about the route can be sent via push notifications to a smartphone. The user clicks on the notification and launches an augmented reality scene that shows him the best route.

\subsection{Visual computing}

Visual computing is a field of computing that deals with the acquisition, analysis, and synthesis of visual data using computer resources. It covers several areas of science, in particular, computer science, mathematics, physics, computer vision, graphics, animation, modelling of complex systems related to visualization, as well as cognitive sciences. Visual computing is used to visualize the results of computer calculations, can help to understand the meaning contained in a huge datasets. Visual computing focuses on the management of activities (for example, business processes in an organization) and interaction through the manipulation of visual virtual images and physical objects [13]. Visual computing is concerned with the creation, processing and analysis of digital images. Images, 3D models, videos, block diagrams and even simple signs can be used as media.

Technologies. In applied research, innovative solutions in the field of data visualization and augmented reality are developed mainly through a combination of computer graphics and computer vision. Visual computing today relies on the processing power of graphics processing units (GPUs). Thanks to the parallel architecture, the GPU is best suited for solving visual computing tasks, providing efficient processing of large amounts of data. Computer vision, image processing, and artificial intelligence are necessary to create the intellect of the object with which the information system interacts - the digital twin, the principle of which is described above.

Application areas. People process information much faster when it is presented visually. Maps and other location illustrations create a powerful entrance into the world of big data and digital transformation, providing a context that can reveal hidden insights, increase efficiency, productivity and profit. As noted above, almost all of the applications of spatial computing need support from visual computing side.

\subsection{Interrelation between spatial and visual computing}

Spatial computing enhances the benefits of visual computing by creating true depth perception in a $360^{\circ}$ user-centred space [10]. Therefore, at the intersection of spatial and visual computing, Immersive Analytics (IA) emerges, which seeks to understand how immersion technologies based on spatial computing can be used to create more intuitive and efficient visual structures supported by visual computing. Interaction provides an overview of the information behind the design, creating a mixture of rendering and spatial computing tasks. Visual computing makes complex data accessible and understandable, spatial computing makes it in its turn useful through data visualization. The synergy of these technologies will enhance the combined potential for obtaining the interpretation of insights.

Spatial computing combines distributed smart sensors with computer vision algorithms, thus mixing the physical and the digital assets to better understand and visualize/display the physical world. Location data can give enterprise access to valuable data to support its digital transformation. Let us say a retailer runs a mobile ad campaign by tracking the number of clicks. At the same time, the POS and online sales data generated during the campaign is recorded. In a traditional data analytics model, these two subsets of data - ad clicks and sales — are isolated. However, if the data is accompanied with [geo] spatial information and displayed on a map, managers can quickly see which ad clicks are 
associated with specific physical stores. The visual reconstruction of shopping behaviour can reveal valuable information, such as the correlation between the number of clicks and the distance to the store, whether online sales were made at home or while commuting. Combining multiple datasets with physical locations creates a rich model for data analysis and an intuitive platform for stakeholders to view and understand large amounts of data. Spatial visual analytics spreads insights into data throughout the enterprise.

\begin{tabular}{|c|c|c|}
\hline & Spatial computing & Visual computing \\
\hline Definition & $\begin{array}{l}\text { Area of computing which is used to create user } \\
\text { interface for sending input information and } \\
\text { manipulating the output from the computer }\end{array}$ & $\begin{array}{l}\text { Area of computing, which deals with the } \\
\text { acquisition, analysis and synthesis of visual data } \\
\text { using computer resources }\end{array}$ \\
\hline Tools & $\begin{array}{l}\text { Software for creating AR apps } \\
\text { Software for developing digital twins }\end{array}$ & $\begin{array}{l}\text { Tools for creating } 2 \mathrm{D} \text { and } 3 \mathrm{D} \text { real-time } \\
\text { visualization } \\
\text { NVIDIA GPU graphics processors }\end{array}$ \\
\hline Functions & $\begin{array}{l}\text { Using the surrounding 3D space as a canvas } \\
\text { for the user interface } \\
\text { Mapping digital and physical locations }\end{array}$ & $\begin{array}{l}\text { Real-time creation of 3D models (3D- } \\
\text { reconstruction) based on data received from sensors } \\
\text { Processing and generation of digital images, } \\
\text { creation, capture, analysis and manipulation of } \\
\text { visual data, 2D-image processing (convolution, } \\
\text { spectral analysis, pattern recognition, object } \\
\text { detection) }\end{array}$ \\
\hline Enables & $\begin{array}{l}\text { Interface for interaction beyond physical } \\
\text { capabilities or as support } \\
\text { Perception of space through AR / VR headsets } \\
\text { Matching human cognitive capabilities } \\
\text { Seamless interaction between people, products, } \\
\text { processes, physical spaces } \\
\text { Understanding how physical space can be used } \\
\text { to improve efficiency and safety }\end{array}$ & $\begin{array}{l}\text { Digitizing data generating visual content } \\
\text { Effective 3D UX design } \\
\text { Using information from cameras and sensors for } \\
\text { people }\end{array}$ \\
\hline $\begin{array}{l}\text { Benefits for } \\
\text { digitalizatio } \\
\qquad \mathrm{n}\end{array}$ & $\begin{array}{l}\text { High operating speed, low training time, } \\
\text { increased productivity, minimized } \\
\text { malfunctions } \\
\text { Optimization of complex work in the } \\
\text { environment by obtaining a complete picture } \\
\text { of movements in space in real time or over a } \\
\text { certain period }\end{array}$ & $\begin{array}{l}\text { Human ability to understand vast and complex } \\
\text { information } \\
\text { Interactive data analysis } \\
\text { Realistic virtual images }\end{array}$ \\
\hline
\end{tabular}

Table 2. Characteristics of spatial and visual computing

This paper focuses on Spatial and Visual computing systems and their interplay in real world ITS applications. It should be noted that specific scenarios from aviation industry discussed further require high-quality visual solutions.

\section{Spatio-visual framework for distributed smart service application}

In this section, we describe and discuss those components of the data processing/analytics pipeline that can be included in the universal integrated spatio-visual framework. All of them serve to solve problems of spatial and visual computing within the enterprise MIS environment. To identify the major components, we use the top-down approach to the design of the framework that implies a stepwise refinement of the required functionality through several subsystem levels until the specification of the base elements (modules) that can provide it. The following subsections provide information about the constituents of our model framework, the interaction between components, and their functionality.

\subsection{Conceptual view of the system}

Location-based analytics are used by leading organizations to address business challenges and unlock new opportunities. Location information is an essential addition to business intelligence (BI) tools. It is important to note that spatio-temporal data requires specific (spatial computing) approaches to its handling and the use of dedicated techniques and tools.

Maps as a core of the geo-spatial data visualization provide meaningful context through which the various participants in the process (providing a service or working in a production) can view specifically the data that is required for their individual roles - and for a broader, common understanding of the entire company. Broadly speaking, maps can serve as an effective spatio-visual framework for organizational integration. 
The user interface, that allows employees and customers interact with information via mobile devices, computers and the IoT devices, maps provide an intuitive picture of work in different sections and planes. Smart maps that have functionality for managing processes can also be called custom Dashboards. Layers of data such as demographics, traffic, and weather can be added to them. With the help of smart maps, organizations can get information about their assets, their condition and location.

Easy-to-use smart maps and spatial analytics provide companies with the confidence to make big, often very costly, decisions. Presenting data visually, so that stakeholders can understand not only what happened, but where and in what context, instantly makes big data more understandable and valuable. By creating and mixing physical and visual context, businesses can better evaluate and make more intelligent use of the vast amounts of information at their disposal. The visual representation of the map is supported by geographic system information (GIS) technology, which serves to manage, visualize, analyse and ultimately understand the importance of location information, obtain actionable information based on the integration of various types of data; CRM information system designed for customer relationship management; business intelligence (BI) tools, as well as systems for managing records, assets and many others.

Below, we provide more details on the components of the suggested application system framework based on spatial and visual computing technologies (Fig.3). Note, the proposed solution features exclusively Open-Source technologybased solutions for versatility.

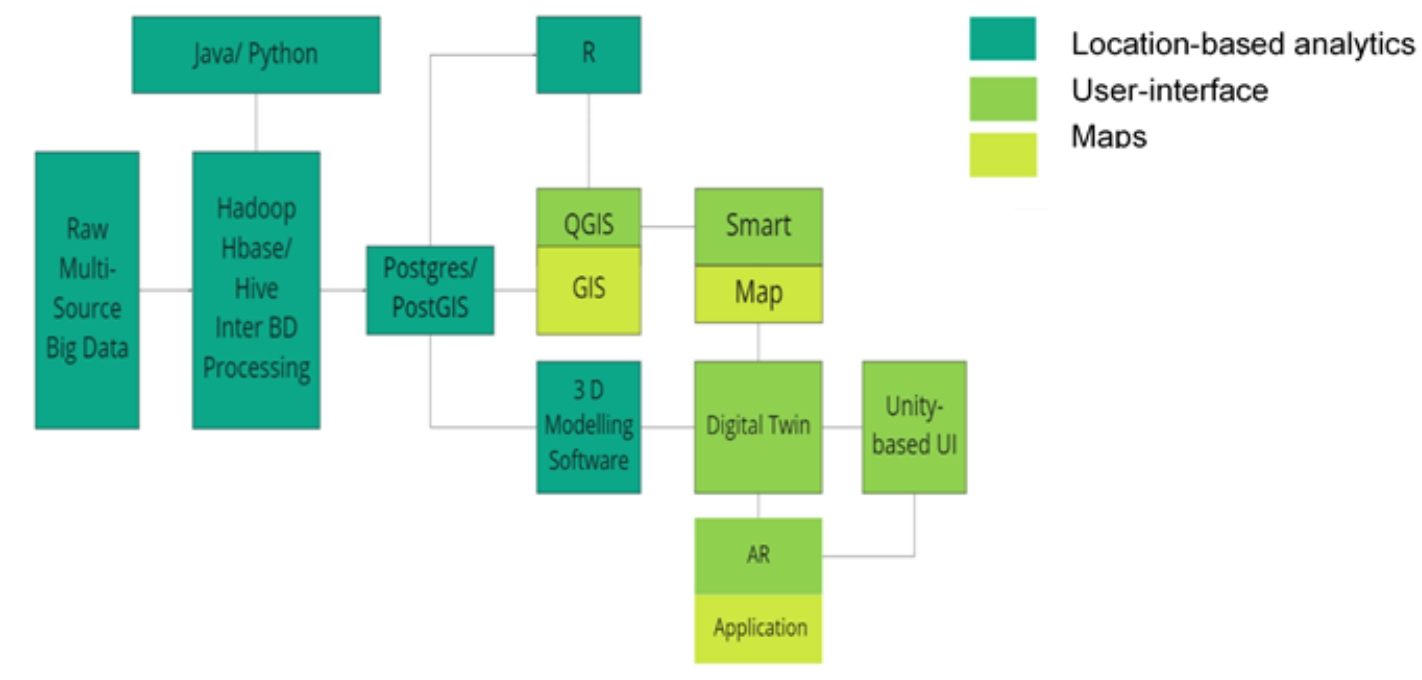

Fig. 3. Block diagram of the integrated spatio-visual application framework.

In terms of architecture, spatial computing provides a great opportunity for scripting, giving a possibility and access to generating plenty of ideas, especially for dynamic spaces such as rooms, buildings, neighbourhoods, cities, and everything that populates these spaces. Spatial computing and augmented reality together enable architecture with an ideal and realistic view of the space available and the "virtual lens" mechanism through which new information can be viewed and applied.

\subsection{Architecting the system}

The application system is architected in a way to provide a web-based user interface to the spatio-temporal analytical and visualization services through ad-hoc APIs and interactive maps.

An important role in the creation of such an application system is the collection of data according to certain principles. It is important to note the nature of the data, in complex objects, which are mainly implied in the writing of this work, both visual and spatial data - Big Geospatial Data. The received data is integrated into the built-in map and then transferred to build an actual 3D model. Binding to geolocation allows restoring the complete picture, objects from the environment, and their representation in three-dimensional space.

Modern open-source technologies such as Big Data technology stack from Apache.org software foundation have potential to handle massive data with high scalability and low latency. Big data computing platforms such as Spark and Hadoop do not offer native support for spatial data. There are Hadoop-based spatial frameworks for big data: Spatial Hadoop and Hadoop GIS, which can become a core part of the Hadoop ecosystem in this project and provide data preprocessing with Hbase and Hive. It is important to note that these frameworks are only capable of performing spatial operations on datasets available in text file formats. Therefore, Python and Java will be used in pre-processing support. 
Spatial database [14] is a database solution for storing and accessing spatial data or data defining geometric space. Spatial databases perform a number of spatial computation operations that can be found in the specification of the standard by The Open Geospatial Consortium (OGC). These include spatial dimensions, spatial functions, spatial predicates, geometry constructors, observer functions. In addition, spatial databases are capable of handling 3D object data, which means that information about each 3D model can be stored in the same database. The diagram shows that among all the alternatives, PostGIS was chosen as an open source library and a spatial database extender for PostgreSQL that runs on top of it and adds spatial datatypes and geometry processing functions for queries.

Once spatial big data becomes available, data analytics tools can provide more information and improve the accuracy of results [15], leveraging the full potential of spatial computing. In this project, we have explored the potential of the data analysis tool provided by R, an OSS environment for statistical calculations and graphics, the programming language has more than 100 available spatial packages, as well as basic geo-visualization tools.

GIS is a tool for processing and visualizing any type of geospatial data. The technology allows users to visualize, analyse, query results, create templates, and view relationships. GIS software contains a variety of applications that combine the use of digital maps and geo-referenced data. QGIS is represented on the proposed model, since this open source software performs all the necessary functions for a project to collect, store, query, analyse, visualize and output data. It can be connected to other systems that support spatial functionality: PostGIS, R, and Python.

The data visualization capabilities of the framework should demonstrate analytical power and dynamic layouts. Applications can be developed for the dashboard generation to display the results of data analysis. The visual analytics implementation can lead to savings in operating costs by more than $20 \%$ due to the reduction of staff time and effective adjustment. GIS-based maps and interactive visualizations can then serve as a communication tool between different teams, departments and geographically distant locations. The Smart Map can act as a platform and interface in which people, space, hardware, software, content, services, IoT devices are integrated. The dashboard can display a planner, calendar, instructions, messages, tools for filtering information and submitting requests. In addition, the digital twin can be accessed through the map interface.

\section{Prototype smart MRO system}

The proposed framework has prospects of application and implementation in the chosen domain of aircraft engine MRO. The section is describing elements of the application project.

\subsection{The smart MRO application framework}

From the operational point of view, the project may contain the following application parts:

1. Dashboard for engine location tracking (users are Managers at OEM's, Airlines Representatives):

- Includes the newest information on condition of the engine

- Allows to observe and monitor the mobility of the engine from airport to airport, from airport to service

- Operative check of the operating status of an aircraft engine

- Role-based reports.

2. Spatial computing systems issues include definition and development of spatial computing infrastructure; collection, integration and storing sensed data; computational aspects of spatial Big Data, and augmented reality:

- Provides guidance for technicians, who are working with the physical object

- Includes the newest information on condition of the engine.

3. Cross-cutting solutions focus on ubiquitous computing arrangements, sustained sensing and monitoring, reliable transportation systems and geo-confidentiality:

- Help to see the engine rendering the complexity of the 3D models.

As the next step, let us take a look at all the parts of the model through the prism of the aviation MRO. The figure 4 shows how Big Spatial Data gets into database at maintenance site. 

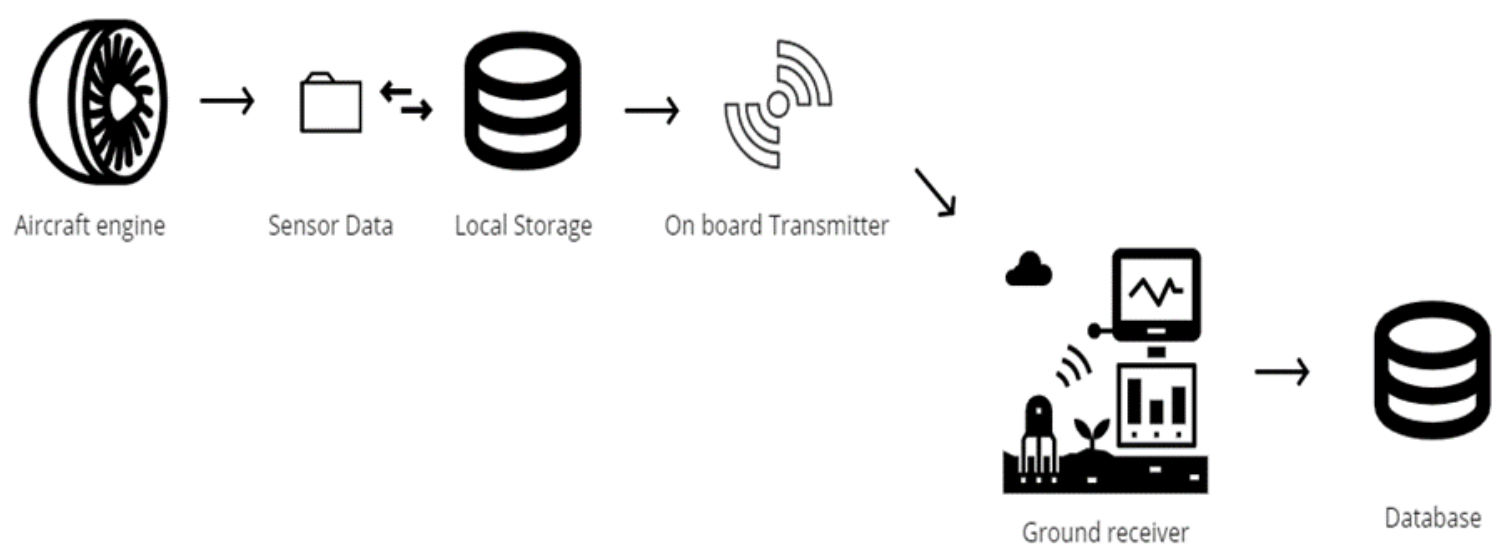

Fig. 4. Aircraft engine data path from data origins to the MRO service database

Coming back to the Figure 3, the type of the database will be essentially the spatial database. Within the scope, spatial databases are capable of handling 3D object data, so that in the same database the information on 3D model of each engine and the information from sensors of an actual engine might be stored and then displayed on the same dashboard, where the locations of engines are presented, too.

Spatial computing can play a significant role as using geo-oriented technologies serving the problem of finding an optimal decision, better orientation in terms of nodes, where are suitable technicians or spare parts, how fast can they reach this place. Analysis based on spatial computing and growing knowledge base will result in building a profound network of all players in this process. Efficient organization of technician's work, who exactly know where and when they have to manage the repair quickly.

The analysis will answer the following questions: who is available and who have right competencies for the task. Flight crewmembers will make qualified decisions; determine the required action that is needed to be done. Suppliers will be on time notified. There will be no need to replace an aircraft if all problems are solved on time.

Use cases of GIS:

- Engine manufacturers are getting knowledge about what is happening in geographical space with their products

- Enabling efficient scheduling of maintenance activities for engines, assigning work to a particular repair team in a particular location, and configuring tasks based on the visualized [geo-] spatio-temporal data.

The Data Visualization framework will show the analytical results with dynamic layouts. A dashboard application will be designed to depict the analytical results. Elements of a dashboard include:

1. Visualize aircraft engine performance parameter: Visualize Efficiency, Visualize Pressure Ratio, etc...

2. Visualize models of aircraft engines: Visualize nominal geometry; Visualize engine.

3. Inspect models: Navigation in 3D Space, Zoom-in / zoom-out / rotate the model.

4. Associate it with particular model: Observe and Compare differences between the nominal (semi-transparent shape) and particular (overlaying shape) geometries

As a result, implementations can lead to savings over $20 \%$ in operational expenses through reduction of staff working time and efficient adjustment. GIS-based maps and visualizations will serve in this case as a communication tool between different teams, departments and geographical far apart from each other locations. Manufacturing directors can visualize everything happening with the products. On the dashboard scheduler, calendar, repair time, needed details, checks from technicians (who checked, who made double check), logs, critical parameters will be mapped. In addition, an access to the digital twin will be realized through the map interface, VR (the whole view of computer-generated picture).

Visual inspection as a predictive/corrective maintenance technology is highly useful [16]. Monitoring for anomaly detection and prevention is an essential work during normal flight and mission execution. With this support, the technicians on site have the opportunity to check the status and position of all planned components and work much more precisely. 
In addition, they can coordinate ad-hoc with component developers in their offices and workshops using collaborative tools. The transfer of the extensive CAD data to the terminal requires high bandwidths - even inside the parked aircraft. Key benefits of using Visual Analytics in maintenance are as follows:

- Visibility of a system status

- Match between the system and the real world

- User control and freedom

- Help users recognize, diagnose errors

- Closeness of mapping

AR Application to MRO is the next idea of the project when an AR application system for a technician is provided to enable him to visually explore the object details through the AR prism. By establishing a connection between the remote expert and the on-site worker, a precise visual guidance can be provided through AR pointers and annotations, displayed on a head-mounted system or a tablet. AR solution will be needed to show 3D model of the engine in real time, allow to get inside a virtual AR cockpit or explore an immersive engine model and to see the working parts from various angles. When user is gazing over an interaction object, which is internally facilitated by the ray tracing, the object highlights itself, that is, the user receives a return feedback signal in the form of a visual clue. As for specific hardware, a device in combination with glasses/head gear will be proposed, as it would be more convenient to have free hands while working. Technicians will fix the event with help of detailed information and required spare parts provided to them to the right time. Thus, maintenance activities are completed faster thus reducing the total Aircraft on Ground (AOG) time. Suitable will be those models of AR glasses that are compatible with Unity3D and transitively with Vuforia that suggest a toolbox for realization of a spatial application.

The benefits of using AR in maintenance are as follows:

- Better ramp handling - ground handling workers can work more efficiently, while they do not have to go through papers or look at tablets

- Faster ramp operations with less errors, for the AR reducing gaps where mistakes used to occur

- More efficient assembling process, because technicians equipped with AR glasses receive instructions in real time with better accuracy. AR headgear gains simple access to engine data (also during the flight)

- Enhanced communication. AR helps users to establish an instant video/audio communication. Expertise can be provided on demand from any remote location

- Convenient Data Access

\section{Discussion}

Spatial computing and visual analytics are used all over the world to discover new information and knowledge from raw data and make informed decisions. Organizations that rely on spatial data analysis represent a wide range of human activities - these are state and local government, national agencies, various businesses, engineering companies, colleges and universities, NGOs. Consequently, this model, which proposes to combine technologies, focused on the features of spatial and visual computing, can have a wide range of applications. For example, the field of aviation [3], namely the technical support of engines that was considered in the work. Aviation industry is facing two major challenges of safety and performance improvement. Business intelligence built within the framework of the proposed model can provide the following: as a result, the application will eliminate the need to replace the aircraft, because decisions on repair and its progress will be applied in a timely manner.

Spatial computing can play a significant role, as the use of geo-oriented technologies serves the task of finding the best solution, finding the best network node with the right technicians or spare parts. Analysis based on spatial computations and a growing knowledge base will create a deep network of all participants in this process. An optimal organization of the work of technicians can be built, who know exactly where and when they have to cope with the repair. The analysis can provide answers to the questions: who is available, and who has the necessary competencies to complete the task.

Flight crewmembers will make informed decisions, determine the actions to be taken, and part suppliers and part manufacturers will be notified in a timely manner. Moreover, cases where spatial computing is used in an industrial plant and several machines and robots need to be connected have to be considered. To work with robots, a large number of sensors are used, and it is necessary to check a huge number of time series with coordinates in the drop-down menus on the desktop, track various data structures.

The framework described in this work may potentially solve the task, by providing the aviation technician with an interface that combines all necessary knowledge based on pre-processed data and information. 


\section{Conclusion}

In this work, the focus of research was made on leveraging technologies of spatial and visual computing for modernization and improvement of aviation MRO efficiency and on new ways of orchestrating them in integrated application framework intended for improvement of the aircraft engine MRO service. In the frames of the work, it was revealed that it is advisable to carry out digital transformation of the transportation business using spatial computing, for example, in cases where advanced personnel interacting with the physical world are involved in business processes. Spatial computing promises to be transformative in a narrower sense and within a targeted set of use cases and verticals (e.g. Smart Health, Smart Transport, and Smart Cities). Spatial analytics add essential context to everything from shopping habits to transportation patterns and industrial service needs. Spatial analysis can combine information from various independent sources and extract qualitatively new information using complex combinations of spatial operations. A comprehensive collection of spatial analysis tools helps to find answers to complex questions related to the organization of working space. Statistical analysis can help to understand whether the observed patterns are meaningful, and visual analysis can reveal changes over time and in real time.

For future research it is planned to adjust the suggested spatio-visual framework to a real industry case. The further steps of the work will be devoted to the creation of a digital twin of an aircraft engine or its core parts and development of a visual analytics tool. Thus, a large part of a future work can be defined as work with data: gathering, transformation, pre-processing, modelling and with implementation of a discussed software. Additionally, analysis of other domains that could benefit from the spatio-visual framework will be conducted.

\section{Acknowledgments}

The reported study was partially supported by RFBR grant No 20-07-00958 and the HSE University Project Group Competition grant in $2020-2022$.

\section{References}

[1] Singh, B. and Gupta, A. (2015) "Recent trends in intelligent transportation systems: a review," Research Directory, J. Transp. Lit. 9 (2), Apr 2015

[2] Peters, J. (2018) "Big data: Unlocking the value of data in air transport", International Airport Review, June 19, 2018. URL: https://www.internationalairportreview.com/article/79124/unlocking-value-data-air-transport/. Accessed 15 July 2021.

[3] Lyu, Y., Liem, R.P. (2020) "Flight performance analysis with data-driven mission parameterization: mapping flight operational data to aircraft performance analysis," Transportation Engineering, 2020.

[4] Brown, R. (2017) “The Connected Fleet: Further Implications of Aircraft Health Monitoring for the Aviation Supply Chain", ICF White Paper, 2017.

[5] Vieira, D.R. and Loures, P.L. (2016) "Maintenance, Repair and Overhaul (MRO) Fundamentals and Strategies: An Aeronautical Industry Overview," 2016.

[6] Chang, H.M. and Abdullah, K. (2014) "The Operation Management Model of Aircraft Maintenance, Repair and Overhaul (MRO) Business,” Int'l J. of Trends in Economics Management \& Technology (IJTEMT), 2014.

[7] Bachan, A. (2019) "How to navigate the complex landscape of emerging MRO technology," ICF, June $27,2019$. URL: https://www.icf.com/insights/transportation/navigating-emerging-mro-technology. Accessed 15 July 2021.

[8] Bachan, A. (2020) "Using digital threads and twins to improve the MRO lifecycle," ICF, March 26, 2020. URL: https://www.icf.com/insights/transportation/improving-mro-lifecycle. Accessed 15 July 2021.

[9] World Economic Forum Top 10 Emerging Technologies of 2020 Special Report, November 2020.

[10] Greenwold, S. (2003) "Spatial Computing", Manuscript submitted to school of architecture and planning, Massachusetts Institute of Technology, Cambridge, June 2003. URL: http://acg.media.mit.edu/people/simong/. Accessed 17 July 2021.

[11] Hashplay Inc. Company Presentation Spatial Computing for Enterprises, June 20, 2017.

[12] Nakamura, T. (2020) "Digital Twin Computing Initiative”, NTT Technical Review, Vol. 18, No. 9, Sept. 2020.

[13] Posada, J. et al. (2015) "Visual Computing as a Key Enabling Technology for Industrie 4.0 and Industrial Internet," in IEEE Computer Graphics and Applications, vol. 35, no. 2, pp. 26-40, Mar.-Apr. 2015, doi: 10.1109/MCG.2015.45.

[14] Advances in Spatial and Temporal Databases: 12th International Symposium, SSTD 2011. Challenge and Vision Track. Springer, 2011.

[15] Gelfand, E. et al. (2010) "Handbook of Spatial Statistics," Chapman and Hall/CRC Handbooks of Modern Statistical Methods (ISBN: 1420072870), 2010.

[16] Katona, A.and Panfilov, P. (2018) "Building Predictive Maintenance Framework for Smart Environment Application Systems," in Proceedings of the 29th DAAAM International Symposium, Vienna, Austria, 2018, pp.0460-0470. DOI: 10.2507/29th.daaam.proceedings.068

[17] Beverungen, D., Müller, O., Matzner, M. et al. Conceptualizing smart service systems. Electron Markets 29, 7-18 (2019). https://doi.org/10.1007/s12525-017-0270-5 